\title{
Design and Validation of the VIDA Questionnaire, for Assessing Instrumental Activities of Daily Living In Elderly People
}

Inaki Martin-Lesende ${ }^{1 *}$, Kalliopi Vrotsou ${ }^{2,3,4}$, Itziar Vergara ${ }^{2,3,4}$, Andoni Bueno $^{5}$, Ana Isabel Diez ${ }^{5}$, Jazmina Nunez ${ }^{5}$, Inmaculada Sanchez C $^{5}$ and Pilar Lopetegui ${ }^{5}$

${ }^{1}$ San Ignacio Primary Care Health Centre, Bilbao-Basurto Integrated Healthcare Organization (IHO), Basque Health Service (Osakidetza), Bizkaia, Spain

${ }^{2}$ Primary Care Research Unit, Gipuzkoa IHOs, Osakidetza, Spain

${ }^{3}$ Health Services Research on Chronic Patients Network (REDISSEC), San Sebastian, Gipuzkoa, Spain

${ }^{4}$ Biodonostia Health Research Institute, Basque Country, Spain

${ }^{5}$ Beraun Primary Care Health Centre, Gipuzkoa IHO, Osakidetza, Spain

\begin{abstract}
The detection of frailty in a community setting requires tools that assess the early stages of functional decline. For this reason, it is important to have adequate scales to assess instrumental activities of daily living (IADL).

The objective of this paper is to provide an overview of the development of a questionnaire (VIDA) for assessing IADL in elderly people in primary care settings and in the community, and its current level of validation.

Using a Delphi study, 10 items were selected for the questionnaire, each with 3- or 4-point Likert scale response options, summing to a total score of 10 to 38 points. No gender bias was detected and the completion time was 3 to 5 minutes. The questionnaire has a high inter- and intrarater reliability, with intraclass correlation coefficients of 0.94 (95\% Cl: 0.88-0.97, p<0.0001) and 0.96 (95\% Cl: 0.93-0.98, p<0.0001), respectively. It has good internal consistency, with a Cronbach's alpha of 0.94 , and the most recent studies suggest that it is moderately sensitivity to change. It also has adequate construct validity, with correlations between items $>0.40$, except for the item "maintaining social relationships" which has a lower factor loading and correlation than the others. The VIDA questionnaire is relatively well correlated with other instruments assessing functioning (Lawton and Brody index and the Timed Up and Go test).
\end{abstract}

Keywords: Validation; Activities of daily living; Questionnaire

\section{Introduction}

Functioning is the ability, generally associated with mobility and social function, to carry out activities that enable an independent life at home and in the community. In fact, the World Health Organization (WHO), in the International Classification of Functioning, Disability and Health (ICF) [1], defines functioning as the positive aspects of the interaction between individuals' health conditions (diseases, disorders, injuries or trauma) and their context (environmental, social and personal factors); and considers that functioning provides a better assessment of the overall health status of elderly people than merely reviewing or listing their health problems. In turn, disability, related to a loss of functioning, seems to be the best predictor of adverse events, deterioration and dependency both at the individual and population level, better even than age or multimorbidity [2]; therefore, it is a key factor to consider in the clinical approach to elderly people, as well as in health policies for this population.

Closely linked to functioning is the concept of frailty. It may precede disability and, if detected early, is potentially reversible. The term frail elderly refers to a person with decreased physiological reserves, who is more likely to have or is more vulnerable to adverse health-related events (hospitalization, falls, post-surgical complications, infection, immobility and other geriatric syndromes, among other problems) and is more likely to experience decline in physical function and functional capacity (disability, dependence) [3,4].

Generally, functioning is evaluated with scales that, in a structured way, assess people's ability to independently perform a series of activities related to their interaction with their environment, so-called activities of daily living (ADL). These ADL are in turn divided into two main groups. On the one hand, there are basic activities of daily living (BADL), which includes self-care tasks (bathing, personal hygiene, grooming, dressing, self-feeding, etc.) and functional mobility (transferring from bed to chair, moving around home, etc.). On the other, there are instrumental activities of daily living (IADL), involving more complex tasks (taking medication, transportation in the community, managing personal finances, using the telephone, etc.). While the former are essential for remaining independent in fundamental functions in one's immediate environment (home), the latter are needed for remaining independent in the community, and require more advanced skills, which tend to be lost earlier [5].

There are well-established scales to assess BADL (e.g., Barthel Index) and others to assess IADL, these being key in relation to frailty and the detection of early stages of functional decline or pre-clinical disability. The Lawton and Brody IADL scale [6] is one of the best-known and most widely used, despite the suggestion of potential cultural and/or gender bias in the results. Nevertheless some research in our setting both refutes this suggestion and demonstrates the usefulness of the scale [7]. Nevertheless, since its development, not many studies have focused on its cross-cultural adaptation and validation. On the other hand, other instruments such as the COOP-WONCA charts [8], the physical function scale of the SF-36, and the Late-Life Function and Disability Instrument $[9,10]$, are not at all widely used, especially in primary care settings.

Another approach to assessing functioning, widely used to detect

*Corresponding author: Inaki Martin Lesende, Centro de Salud de San Ignacio C/Larrako Torre, 948015 Bilbao, España, Spain, Tel: +34 946006692; Fax: +34 946006693; E-mail: inaki.martinlesende@osakidetza.eus

Received March 27, 2015; Accepted April 27, 2015; Published April 29, 2015

Citation: Martin-Lesende I, Vrotsou K, Vergara I, Bueno A, Diez Al, et al. (2015) Design and Validation of the VIDA Questionnaire, for Assessing Instrumental Activities of Daily Living In Elderly People. J Gerontol Geriat Res 4: 214 doi:10.4172/2167-7182.1000214

Copyright: (c) 2015 Martin-Lesende, et al. This is an open-access article distributed under the terms of the Creative Commons Attribution License, which permits unrestricted use, distribution, and reproduction in any medium, provided the original author and source are credited. 
frailty, is to carry out functional mobility tests. These tests are simple to perform, and the results are well correlated with those of functional assessment questionnaires. Moreover, they are not influenced by cultural factors and can potentially predict physical decline and disability. In such tests, participants perform specific tasks, generally involving gross motor skills including walking or related to balance or limb mobility. Three of the most widely-used and recognized tests are the Gait Test [11], the Timed Up and Go test and the Short Physical Performance Battery (SPPB) [12].

The urgent need for standardized and valid tools for assessing functioning and IADL, which are key for detecting the onset of frailty, was the driver behind the creation and validation of the VIDA questionnaire, the acronym being derived from the Spanish for daily life of the elderly person ("VIda Diaria del Anciano"). This questionnaire was developed around the following criteria: it should be focused on elderly people in the community, be easy to use in routine primary care practice, be able to detect the onset of a decline in functioning and provide a multidisciplinary perspective.

In this paper, the stages of the development and validation to date of the VIDA questionnaire are presented, for assessing the IADL of elderly people in a community setting.

\section{Materials and Methods}

Figure 1 summarizes the successive steps being followed for the development and validation process of the VIDA questionnaire. We started in November 2003 with a Delphi study, and then conducted reliability analysis. Currently, we are analyzing the data from the use of the questionnaire in a cohort study (KOSFRAGIL)in which a tool for the identification of frail elderly in primary care settings is proposed.

Initial Delphi study [13], to select items for the questionnaire (content validity of the instrument based on expert criteria): 57 experts from a wide range of professional groups participated in the study (general practitioners, geriatricians, nurses, physiotherapists, and social workers) identified though the Spanish Society of Family and Community Medicine (semFYC), and the Spanish Society of Geriatrics and Gerontology (SEGG). Three consecutive rounds of surveys were sent to participants by e-mail (or fax, on request). In the first round, participants were asked what items should be included on a questionnaire to assess IADL in elderly people; in the second round, they were asked to select, from various groups of items, the 10 they felt were the most important; and in the third and last round, they were asked to rate, on a scale of 1 to 10 , the importance of the 14 items most commonly selected in the previous round. In this way, considering the item ratings, we decided on the items to include in the initial version of the instrument.

Having produced a draft questionnaire, we asked two experts in questionnaire design and methodology to review it, and later preliminary pilot testing was carried out with consensus meetings [14]. In this way, we ensured that the questionnaire had apparent validity.

Analysis of inter- and intrarater reliability [15], by administering 71 pairs of VIDA questionnaires to people $\geq 75$ years old without significant sensory, physical or mental impairment. We consecutively recruited participants by stratified sampling of individuals with different degrees of impairment in BADL (total Barthel Index scores of $>90$ points, indicating independence or only slight dependence, $42 \%$; $61-90$ points, moderate dependence, $42 \%$; and $<61$ points, severe dependence, $16 \%$ ). We also explored the presence of other factors related to frailty (recent hospitalization, comorbidity, polypharmacy, etc.) and their correlation

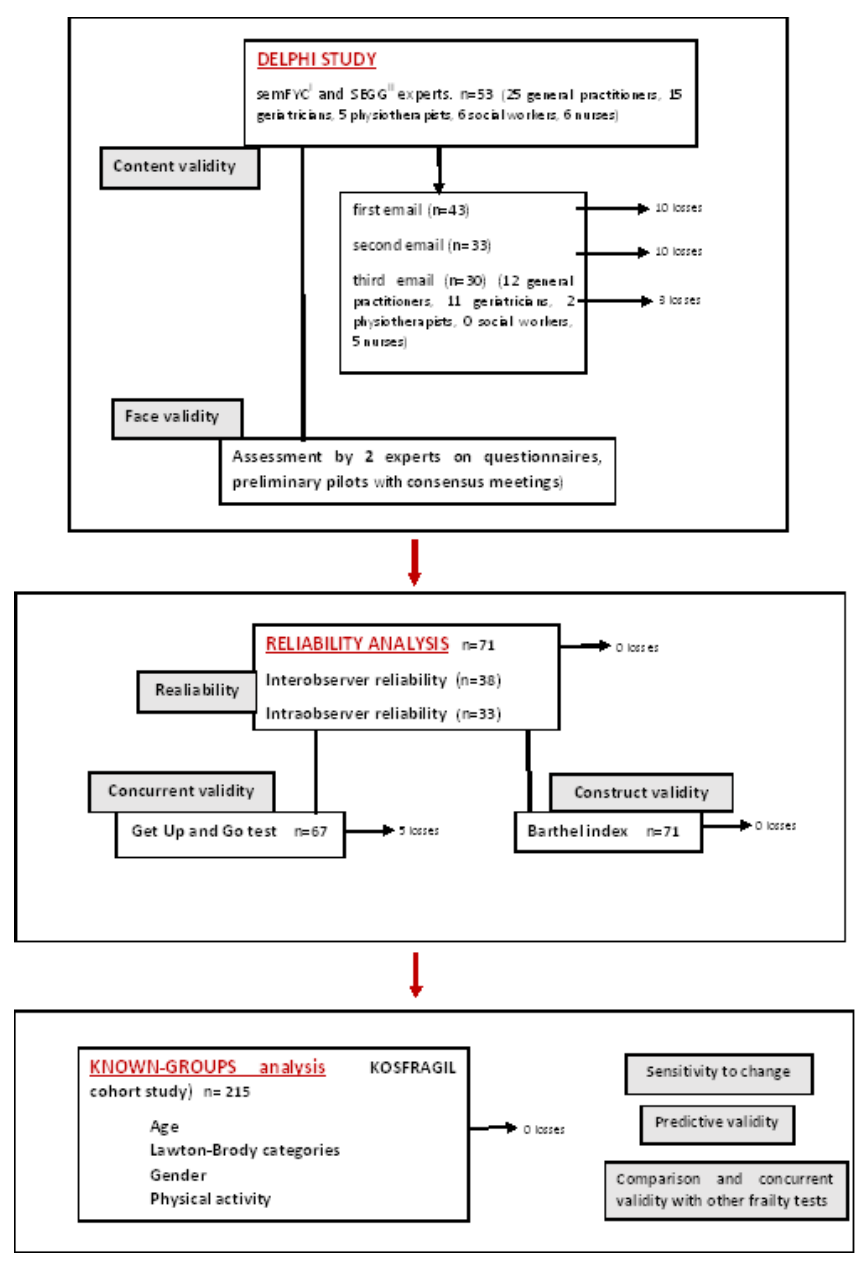

Figure 1: Phases of the development and validation of the VIDA questionnaire

I semFYC: Spanish Society of Familiy and Community Medicine

II Spanish Society of Geriatrics and Gerontology

with the Barthel Index scores [15], as well as the concurrent validity with the Timed Up and Go test.

Subsequently, we continued the validation process by performing exploratory factor analysis (to assess construct validity) [16] in the same sample used previously for the reliability analysis. We used the un weighted least squares estimation, given the categorical nature of the responses, and factor loadings and communalities $\geq 0.40$ were deemed acceptable. We also analyzed Spearman's correlations between scores on different items and between scores on each item and the total scale excluding that item [16].

Analysis of known-group validity, considering age group $(\leq 80 / 81$ $85 />85$ years old), Lawton IADL scores (0-4/5-8 points), level of physical activity (low/high), and gender (male/female). This analysis was performed with 215 individuals $\geq 75$ years of age from the cohort KOSFRAGIL who were independent on recruitment to the study.

Based on the analysis of the KOSFRAGIL cohort, we have already obtained data on sensitivity to change of the VIDA questionnaire. We are currently assessing the predictive validity (for adverse events) and making comparisons with other indices and tests used for detecting frailty. 


1. PREPARING AND TAKING MEDICATIONS (repeat and on-off prescriptions)
- Obtains, prepares, and takes drugs without help
- Others obtain, supervise or record when he/she should take them
- Takes drugs others have prepared/left ready
- Others take care of everything, even giving him/her each dose
2. USING THE TELEPHONE
- Makes and answers calls with no difficulties
- Answers calls, but only makes calls to certain numbers
- Only answers calls
- Is not able to use the telephone
3. CARRYING OUT HOUSEWORK AND OTHER DOMESTIC TASKS
- Complex tasks (with large appliances, iron, cooker, drill, plugs, etc.)
- Routine tasks (cleaning, tidying, vacuuming, making beds, changing lightbulbs,
tightening screws, etc.)
- Only simple tasks (sweeping, putting things away)
- None
4. MANAGING FINANCES
- All the domestic finances
- Helps with domestic finances and banking
- Only simple expenses and purchases
- Does not handle money
- Only goes out with assistance
- Walks without assistance
- Uses a wheelchair
-

\begin{tabular}{l} 
6. USING UTILITIES SAFELY \\
- Routine tasks (with gas, electricity, e.g., cooker, water, etc.) without incident \\
- Only simple tasks (e.g., turning on/off taps, switches, etc.) \\
- None of the above \\
7. DOING THE SHOPPING \\
- Any type of purchase \\
- Simple purchases \\
- Needs help to shop \\
- None \\
8. USING DOORS \\
- Opens and closes doors with keys \\
- Opens and closes doors with handle or knob, but not with keys \\
- Unable to open or close doors \\
9. USING TRANSPORT \\
- Makes any journey or drives herself/himself \\
- Needs help or someone to accompany her/him for non-routine or long journeys \\
- Always needs someone to accompany her/him \\
- Only travels in special vehicle or ambulance \\
- Socialises with friends and family \\
- Only socialises with family \\
- Does not socialise \\
MAINING SOCIAL RELATIONSHIPS \\
- Total score: \\
\hline
\end{tabular}

Figure 2: VIDA questionnaire

\section{Results}

We selected the 10 items with the highest ratings in the Delphi process, the remaining items having obtained markedly lower scores [13]. After the assessment by the two experts and the preliminary pilot testing of the draft, we established the questionnaire to be used in subsequent studies (Figure 2). Items are rated on 3- or 4-point Likert scales, making it possible to assess changes in an individual over time in each item, and sum to a total score ranging from 10 to 38 . The completion time is between 3 and 5 minutes, once evaluators are familiarized with the scale [14].

In the reliability study, the intraclass correlation coefficients (ICCs) were 0.94 (95\% CI: 0.88-0.97, p<0.0001) and 0.96 (95\% CI: 0.93-0.98, $\mathrm{p}<0.0001$ ) for inter- and intrarater reliability, respectively [15]. A lower score was obtained on the questionnaire when patients had two or more risk factors for frailty $(\mathrm{p}=0.015)$ [15].

With the same sample as the reliability study [15], the VIDA questionnaire was moderately correlated with the Timed Up and Go test (ICC: $0.61, \mathrm{p}<0.0001$ ). We also found that a score of 35 was the cut-off point that gave the strongest association between the questionnaire and the Timed Up and Go test, considering a time of more than $20 \mathrm{~s}$ in this test to be abnormal (indicating problems with gait or balance and hence a risk of falls). That is, having a score $<36$ in the VIDA questionnaire was associated with an abnormal Timed Up and Go test ( $>20 \mathrm{~s})$.

Considering all 10 items, the internal consistency of the questionnaire, assessed using Cronbach's alpha, was 0.94 [16]. The factor loadings were above 0.70 for the first 9 items and 0.47 for item 10 ("maintaining social relationships"). Correlations between items ranged from 0.33 to 0.77 , those with item 10 being the weakest. On the other hand, correlations between items and the total scale were above 0.40 in all cases [16].

The known-group analysis (Table 1) demonstrated that the VIDA questionnaire scores did not contain a gender bias ( $\mathrm{p}=0.418$ between men and women) and that they were associated with the Lawton IADL scale scores, categorized into $0-4$ or $5-8(\mathrm{p}<0.0001)$. Further, scores fell with increasing age $(\mathrm{p}=0.0002)$ and decreasing physical activity $(\mathrm{p}=0.0001)$.

\section{Discussion}

We have developed a comprehensive scale for the assessment of IADL that is easy to administer (taking 3 to 5 minutes) in a primary care setting. Indeed, in terms of the activities assessed, the questionnaire has a greater scope than other tools and includes some novel items, for example, the ability to open/close doors or maintain social relationships. The inclusion of these innovative items reflects the multidisciplinary 


\begin{tabular}{|c|c|c|c|c|c|}
\hline & Mean (SD') & Median (Q1, Q3ii) & $\begin{array}{l}\text { Minimum- } \\
\text { maximum }\end{array}$ & $\mathbf{n}$ & Piii \\
\hline \multicolumn{5}{|l|}{ Age } & 0.0002 \\
\hline$<80$ years old & $35.1(3.1)$ & $36(34,37)$ & $23-38$ & 146 & \\
\hline $81-85$ & $34.5(2.9)$ & $35(33,36)$ & $26-38$ & 46 & \\
\hline$>85$ & $31.9(4.9)$ & $33(30,36)$ & $19-38$ & 23 & \\
\hline \multicolumn{5}{|c|}{ Lawton-Brody index } & $<0.0001$ \\
\hline $0-4$ points & $31.4(4.4)$ & $32(29,35)$ & $19-37$ & 41 & \\
\hline 5-8 points & $35.4(2.6)$ & $36(35,37)$ & $25-38$ & 174 & \\
\hline \multicolumn{5}{|c|}{ Low physical activity } & 0.0001 \\
\hline No & $35.2(2.8)$ & $36(34,37)$ & $21-38$ & 193 & \\
\hline Yes & $30.3(4.8)$ & $30(28,35)$ & $19-37$ & 22 & \\
\hline \multicolumn{5}{|l|}{ Gender } & 0.418 \\
\hline Male & $34.4(3.1)$ & $35(33,37)$ & $25-38$ & 79 & \\
\hline Female & $34.8(3.6)$ & $36(34,37)$ & $19-38$ & 136 & \\
\hline
\end{tabular}

iSD: standard deviation; i"Q1: first quartile, Q3: third quartile; iiistatistical significance through Jonckheere-Terpstra test for the age, t-test for Lawton-Brody index, for physical activity and for gender.

Table 1: Results of known-groups of the VIDA questionnaire.

nature of the expert group involved in the Delphi study [13]. Although the questionnaire includes two items that could be expected to be influenced by gender, "doing the shopping" and "housework", widening the range of activities covered by these items avoids this potential effect, as confirmed in the initial pilot studies in which the questionnaire was adapted until it obtained apparent validity. Specifically, "shopping" includes some tasks often carried out by men, such as buying a newspaper and "housework" extends beyond traditional homemaking tasks to minor home maintenance. The known-groups analysis also found no gender bias in the overall score.

In the reliability study [15], we found very good inter- and intrarater reliability. The ICCs are even higher than those reported by other authors for widely-used and well-established questionnaires assessing BADL functioning, such as Barthel's index [17,18], and those assessing cognitive impairment, such as the Cognitive Mini-Exam (a Spanish adaption of the Mini-Mental State Examination developed by Folstein et al.) [19].

Moreover, the fact that scores in the VIDA questionnaire were poorer when patients had two or more frailty risk factors in reliability study sample (important comorbidity, falls, recent hospitalisation, visual or hearing impairment, polypharmacy, adverse social factors) supports the construct validity of the VIDA questionnaire, in that such individuals with these factors are more likely to experience adverse events and decline towards disability [15]. Consistent with this, in the known-groups analysis, poorer scores in the questionnaire were associated with older age and lower physical activity. Further, the scores were closely associated with those of the Lawton and Brody scale.

Considering all 10 items, there internal consistency was 0.94 [16]. In the analysis of factor loadings and correlations between items and between items and the overall results were high, although lower for the item "maintaining social relationships". These results indicate adequate construct validity, although the aforementioned item seemed to behave differently [16]. Also, this specific question is more complex and difficult to achieve; numerous extensive questionnaires explore it, but it's a challenge to include it as another IADL. Future validation and modifications should explore these characteristics; even considering changing the way social relationships are dealt with, assessing the influence of the item on the results and its formulation in the overall questionnaire.

One limitation to take into account is the fact that in studies based on people without significant impairment in BADL $[14,16]$, scores in VIDA questionnaire tend to cluster at the high end of the scale. Further, in the preliminary analysis of the KOFRAGIL cohort study, the questionnaire was found to have a moderate sensitivity to change. This may be an issue in validation studies and should be anticipated.

On the other hand, although a score below 36 points is associated with poor performance in the Timed Up and Go test, we need to wait for the predictive validity analyses from the KOSFRAGIL cohort study to establish the best cut-off points. In any case, for longitudinal assessments, decreases in the score when repeated over time probably should be noted regardless of the score.

As well as some important analysis of data from the cohort study, pending work includes the evaluation of results of teams that have started using the questionnaire in research and daily clinical practice. We consider the validation to be an ongoing process, and that further research may lead to changes to optimize the questionnaire.

\section{Conclusions}

The VIDA questionnaire is an easy-to-administer tool for assessing IADL in elderly people in a primary care setting and in the community.

It has high reliability and internal consistency.

It also has good construct validity and the scores correlate well with the results of other instruments assessing functioning (Lawton IADL index and the Timed Up and Go test).

A score of less than 36 points is associated with an abnormal Timed Up and Go test, although we should wait for confirmation of its characteristics from the ongoing predictive validity study to establish the optimal cut-offs for IADL impairment.

\section{Acknowledgements}

This research project is currently funded by a grant from the Department of Health of the Government of the Basque Country (ref: 2011111078).

\section{References}

1. ICF International Classification of Functioning, Disability andHealth (2001) Geneva: World Health Organization.

2. Abizanda P, Romero L, Sánchez-Jurado PM, Martínez-Reig M, Alfonso-Silguero SA, et al. (2014) Age, frailty, disability, institutionalization, multimorbidity or comorbidity. Which are the main targets in older adults? J Nutr Health Aging 18: $622-627$

3. Rodríguez-Mañas L, Féart C, Mann G, Viña J, Chattergi S, Chodzko-Zajko W, et al. (2013) Searching for an Operational Definition of Frailty: A Delphi Method Based Consensus Statement. The Frailty Operative Definition-Consensus Conference Project. J Gerontol A BiolSci Med Sci 68: 62-7

4. Consensus document on frailty and falls prevention among the elderly (The prevention and health promotion strategy of the Spanish NHS) (2014) General Sub-Directorate of Health Promotion and Epidemiology. General Directorate of Public Health, Quality and Innovation Ministry of Health, Social Services and Equality.

5. Jette AM (1999) Disentangling the process of disablement. Soc Sci Med 48 471-472.

6. Lawton MP, Brody EM (1969) Assessment of older people: self-maintaining and instrumental activities of daily living. Gerontologist 9: 179-186.

7. Vergara I, Bilbao A, Orive M, Garcia-Gutierrez S, Navarro G, et al. (2012) Validation of the Spanish version of the Lawton IADL Scale for its application in elderly people. Health Qual Life Outcomes 10: 130

8. Nelson E, Wasson J, Kirk J, Keller A, Clark D, et al. (1987) Assessment of function in routine clinical practice: description of the COOP Chart method and preliminary findings. J Chronic Dis 40 Suppl 1: 55S-69S. 
Citation: Martin-Lesende I, Vrotsou K, Vergara I, Bueno A, Diez Al, et al. (2015) Design and Validation of the VIDA Questionnaire, for Assessing Instrumental Activities of Daily Living In Elderly People. J Gerontol Geriat Res 4: 214. doi:10.4172/2167-7182.1000214

9. Haley SM, Jette AM, Coster WJ, Kooyoomjian JT, Levenson S, et al. (2002) Late Life Function and Disability Instrument: II. Development and evaluation of the function component. J Gerontol A Biol Sci Med Sci 57: M217-222.

10. Simonsick EM, Kasper JD, Guralnik JM, Bandeen-Roche KJ, Ferrucci L, et al. (2001) Severity of upper and lower extremity functional limitation: scale development and validation with self-report and performance-based measures of physical function. J Gerontol B Bio Sci Med Sci 56B: S10-S19

11. Castell MV, Sánchez M, Julián R, Queipo R, Martín S, et al. (2013) Frailty prevalence and slow walking speed in persons age 65 and older: implications for primary care. BMC Fam Pract 14: 86.

12. da Câmara SM, Alvarado BE, Guralnik JM, Guerra RO, Maciel AC (2013) Using the Short Physical Performance Battery to screen for frailty in young-old adults with distinct socioeconomic conditions. Geriatr Gerontol Int 13: 421-428.

13. Martín-Lesende I, Ortiz-Lebaniegos I, Montalvillo-Delgado E, Pérez-Abad M, Sánchez-Junquera P, et al. (2006) [Identification of items for creating a questionnaire for the assessment of instrumental activities of daily living (IADL) in elderly patients]. Aten Primaria 37: 313-318.

14. Martín-Lesende I, Valle N, Arnáiz de las Revillas JM, Montalvillo E, Martín LC, et al. (2010) Creación y validación de un cuestionario para valorar actividades instrumentales de la vida diaria en ancianos. Salud (i) Ciencia $17: 638-41$

15. Martín-Lesende I, Quintana S, Urzay V, Ganzarain E, Aguirre T, Pedrero JR (2012) Fiabilidad del cuestionario VIDA, para valoración de Actividades Instrumentales de la Vida Diaria (AIVD) en personas mayores. Aten Primaria $44: 309-19$.

16. Martín Lesende I, Vrotsou K, Vergara Micheltorena I, Bueno Errandonea A (2014) [Exploratory factor analysis of the VIDA questionnaire for the evaluation of the instrumental activities of daily living]. Aten Primaria 46: 317-319.

17. Collin C, Wade DT, Davies S, Horne V (1988) The Barthel ADL Index: a reliability study. Int Disabil Stud 10: 61-63.

18. Roy CW, Togneri J, Hay E, Pentland B (1988) An inter-rater reliability study of the Barthel Index. Int J Rehabil Res 11: 67-70.

19. Lobo A, Ezquerra J, Gómez Burgada F, Sala JM, Seva Díaz A (1979) [Cognocitive mini-test (a simple practical test to detect intellectual changes in medical patients)]. Actas Luso Esp Neurol Psiquiatr Cienc Afines 7: 189-202. 